\title{
CORRIGENDUM
}

\section{Spectrum of CLCN1 mutations in patients with myotonia congenita in Northern Scandinavia}

\author{
Chen Sun, Lisbeth Tranebjærg, Torberg Torbergsen, Gösta Holmgren and \\ Marijke Van Ghelue
}

European Journal of Human Genetics (2010) 18, 264; doi:10.1038/ejhg.2009.217

Correction to: European Journal of Human Genetics (2001) 9, 903-909. doi:10.1038/sj.ejhg.5200736

One of the mutations described in this paper was named incorrectly. The nucleotide and protein codes are correct, but the coordinate is wrong. The corrected Figure $2 \mathrm{a}$ is shown below. Accordingly, F287S (p.Phe287Ser) has to be replaced by F307S (p.Phe307Ser). The consensus sequence (bottom line) has been removed. The authors would like to apologise for the mistake.

\begin{tabular}{|c|c|c|}
\hline & F307S & A331T \\
\hline & 294 & 320 \\
\hline ClC-1Human & STYFAVRNYWRGFFAATFSAFVFRV & AVWNKDAV. . TITALFRTNFRMDFPFD \\
\hline Rat & STYFAVRNYWRGFFAATFSAFVFRV & AVWNKDAV . TITALFRTNFRMDFPFD \\
\hline ClC-2Human & STFFAVRNYWRGFFAATFSAFIFRV & AVWNKDAV . . TITALFRTNFRMDFPFD \\
\hline Rat & STFFAVRNYWRGFFAATFSAFIFRV & AVWNKDAV . TITALFKTRFRLDFPFD \\
\hline Rabbit & STFFAVRNYWRGFFAATFSAFIFRV & AVWNKDAV . TITALFKTRFRLDFPFD \\
\hline ClC-3Human & SYYFPLKTLWRSFFAALVAAFVLRS & NPFGNSR . . . . LVLFYVEYHT . . PWY \\
\hline Mouse & SYYFPLKTLWRSFFAALVAAFVLRS & NPFGNSR . . . . LVLFYVEYHT . . PWY \\
\hline Rat & SYYFPLKTLWRSFFAALVAAFVLRS & NPFGNSR . . . . LVLFYVEYHT . . PWY \\
\hline ClC-4Human & SYYFPLKTLWRSFFAALVAAFTLRS & NPFGNSR . . . . LVLFYVEYHT . . PWY \\
\hline Mouse & SYYFPLKTLWRSFFAALVAAFTLRS & NPFGNSR . . . . LVLFYVEYHT . . PWY \\
\hline Rat & SYYFPLKTLWRSFFAALVAAFTLRS & NPFGNSR . . . . LVLFYVEYHT . . PWY \\
\hline ClC-5Human & SYYFPLKTLWRSFFAALVAAFTLRS & NPFGNSR . . . . LVLFYVEFHT . . PWH \\
\hline Rat & SYYFPLKTLWRSFFAALVAAFTLRS & NPFGNSR . . . . LVLFYVEFHT . . PWH \\
\hline
\end{tabular}

